\title{
Molecular cloning and characterization of the pseudorabies virus $U L 31$ gene
}

\author{
M.L. Li ${ }^{1,2}$, Z.Y. Zhao ${ }^{1}$, W. Cui ${ }^{1}$, C.C. Mo ${ }^{1}$, J.L. Wang ${ }^{1}$ and M.S. Cai ${ }^{1,2}$ \\ ${ }^{1}$ Department of Pathogenic Biology and Immunology, \\ School of Basic Science, Guangzhou Medical University, Guangzhou, China \\ ${ }^{2}$ Institute of Immunology, Sino-French Hoffmann Institute, \\ School of Basic Science, Guangzhou Medical University, Guangzhou, China \\ Corresponding author: M.S. Cai \\ E-mail: mingshengcai@hotmail.com
}

Genet. Mol. Res. 13 (1): 1832-1847 (2014)

Received January 2, 2013

Accepted August 13, 2013

Published March 17, 2014

DOI http://dx.doi.org/10.4238/2014.March.17.11

\begin{abstract}
We amplified a 816-bp sequence of the UL31 gene from the pseudorabies virus (PRV) Becker strain genome. Evidence that this was the UL31 gene was confirmed by cloning and sequencing. The PRV UL31 gene encodes a putative protein of 271-amino acid residues, which was designated the UL31 protein. Bioinformatic analysis indicated that PRV UL31 contains a conserved PHA03328 domain, closely related with the herpes virus nuclear egress lamina protein UL31 family and highly conserved among counterparts encoded by herpes UL31 genes. Nucleic acid sequence and amino acid sequence alignments demonstrated that PRV UL31 has a relatively higher homology with UL31 homologous proteins of subfamily Alphaherpesvirinae than other subfamilies. In addition, phylogenetic analysis showed that PRV UL31 has a close evolutionary relationship with members of the subfamily Alphaherpesvirinae, especially bovine herpesvirus 1 (BoHV-1), BoHV-5, equine herpesvirus 4 (EHV-4), EHV-9 and EHV-1. Antigen prediction demonstrated that several
\end{abstract}


potential B-cell epitopes are located in PRV UL31. Additionally, secondary structure and three-dimension structure prediction revealed that PRV UL31 predominantly consists of $\alpha$-helix. Taken together, these results provide insight on the function and mechanism of UL31 during PRV infection.

Key words: Pseudorabies virus; UL31; Cloning; Bioinformatic analysis; Molecular characterization

\section{INTRODUCTION}

Aujeszky's disease, which is caused by pseudorabies virus (PRV), is a fatal disease with a global distribution that affects swine primarily and other domestic and wild animals incidentally. PRV belongs to the genus Varicellovirus of the subfamily Alphaherpesvirinae, the neurotropic nature of which makes it a useful tool to trace circuits in the mammalian nervous system. PRV is also a useful model for the study of herpesvirus pathogenesis. While efforts to eradicate PRV in many countries have shown great progress, it is still an endemic problem in many countries (Pomeranz et al., 2005).

To study the fundamental mechanisms underlying the spread and pathogenesis of $\mathrm{PRV}$, it is particularly important to gain a comprehensive understanding of the functions of each gene and their products in the course of viral replication. During the lytic cycle of infection, PRV genes are expressed as for other herpesviruses in a sequential cascade manner as follows: immediate-early, early and late. The UL31 protein, which is the product of the UL31 gene, is the component of the primary tegument expressed with late kinetics during PRV infection (Granzow et al., 2004). Herpes simplex virus 1 (HSV-1) UL31 (Chang and Roizman, 1993; Ye and Roizman, 2000; Reynolds et al., 2001; Fuchs et al., 2002; Simpson-Holley et al., 2004; Liang and Baines, 2005; Kato et al., 2006; Mou et al., 2009; Roberts and Baines, 2011; Yang and Baines, 2011) and Epstein-Barr virus (EBV) BFLF2 (Lake and Hutt-Fletcher, 2004; Gonnella et al., 2005; Granato et al., 2008), the homologue of PRV UL31, have been extensively studied, but the function of PRV UL31 is less well understood.

In the present study, the UL31 gene was amplified from the PRV Becker strain genome using polymerase chain reaction (PCR) and then subjected to cloning and sequencing. Subsequently, a comprehensive bioinformatic analysis was conducted to study the molecular characteristics of UL31 to provide molecular biology insight for further study on the function and mechanism of UL31 during PRV infection by employing a large number of bioinformatic tools such as ORF Finder, Conserved Domains, DNAstar 7.0, Bioedit 7.0, SignalP-4.0, NetPhos 2.0, PSIpred, CPHmodels 3.2, etc.

\section{MATERIAL AND METHODS}

\section{Cloning and sequencing of the PRV UL31 gene}

Based on computer-assisted analysis, the primers for PCR amplification of UL31 (accession No. JF797219) were designed by the biological Oligo 6.0 and Primer 5.0 software and 
were synthesized by TaKaRa (Dalian, China). The upstream primer 5'-TTGAATTCATGTTT GAGCGACGGCGGCT anneals with the first 20 nucleotides of $U L 31$ and introduces an upstream EcoRI (underlined) site for cloning. The downstream primer 5'-TGAAGCTTTCACG GGCGAGGGGGGCGAAA is complementary to the final 21 nucleotides of UL31 and introduces a HindIII (underlined) site.

The UL31 gene was amplified via PCR by KOD-Plus-Neo (Toyobo) from the genome DNA of the PRV Becker strain, using the genome previously purified from PRV-infected PK15 cells (Smith and Enquist, 2000; Li et al., 2011a,b) as the template. PCR profiles involved predenaturation for $5 \mathrm{~min}$ at $95^{\circ} \mathrm{C}$ followed by 30 cycles of denaturation at $94^{\circ} \mathrm{C}$ for $40 \mathrm{~s}$, annealing at $62^{\circ} \mathrm{C}$ for $45 \mathrm{~s}$, and extension at $72^{\circ} \mathrm{C}$ for $50 \mathrm{~s}$. A final extension step was performed at $72^{\circ} \mathrm{C}$ for $10 \mathrm{~min}$. The PCR product was then analyzed on a $1 \%$ agarose gel. The purified PCR product was digested with EcoRI and HindIII restriction enzymes and ligated into the correspondingly digested prokaryotic expression vector pET28a $(+)$ (Novagen, Madison, WI, USA), to generate $\mathrm{pET} 28 \mathrm{a}(+)-\mathrm{UL} 31$, and the presence of the appropriate insert in the obtained plasmid was then verified by PCR, restriction analysis and sequencing.

\section{Bioinformatic analysis of the nucleotide sequence of PRV UL31}

To search the nucleotide sequence similarity and open reading frame (ORF), NCBI BLASTN tool (http://www.ncbi.nlm.nih.gov/BLAST/) and ORF Finder (http://www.ncbi.nlm. nih.gov/gorf/gorf.html) were applied, respectively. Subsequently, Clustal V in the MegAlign program of DNAStar (version 7.0, DNAStar, Inc.) (Burland, 2000; Li et al., 2012) was employed to conduct the nucleotide sequence homology analysis of 46 UL31-like proteins of herpesviruses (listed in Table 1).

\section{Bioinformatic analysis of the amino acid (aa) sequence of PRV UL31}

For aa sequence comparison, homology search and conserved domain analysis, the aa sequence of PRV UL31 was analyzed using BLASTP (http://www.ncbi.nlm.nih.gov/ BLAST/) (Altschul et al., 1997) and Conserved Domains search tool (http://www.ncbi.nlm. nih.gov/Structure/cdd/wrpsb.cgi) (Marchler-Bauer et al., 2005), respectively. To compare sequences between PRV UL31 and UL31-like proteins of other herpesviruses (listed in Table 1), aa sequence homology and phylogenetic relationship were analyzed by employing DNAstar 7.0 or ClustalX 1.8 (Thompson et al., 1997). For predicting the signal peptide sequence, transmembrane domain, glycosylation site, phosphorylation site, hydrophobicity and hydrophilicity regions, B-cell epitope, secondary structure, and three-dimensional (3-D) structure, SignalP-4.0 Server (http://www.cbs.dtu.dk/services/SignalP/) (Nielsen et al., 1997; Bendtsen et al., 2004), TMHMM program (http://www.cbs.dtu.dk/services/ TMHMM/) (Moller et al., 2001), NetNGlyc 1.0 program (http://www.cbs.dtu.dk/services/ NetNGlyc/), NetPhos 2.0 program (http://www.cbs.dtu.dk/services/NetPhos/) (Blom et al., 1999), Bioedit 7.0 software (Hopp and Woods, 1981; Kyte and Doolittle, 1982), DNAstar 7.0 software (Van Regenmortel, 1992; Burland, 2000), PSIpred program (http://bioinf. cs.ucl.ac.uk/psipred/) (Jones, 1999) and CPHmodels 3.2 Server (http://www.cbs.dtu.dk/ services/CPHmodels/) were used, respectively. 
Table 1. Abbreviations and accession Nos. of $49 U L 31$ gene products from different species.

\begin{tabular}{|c|c|c|c|}
\hline Subfamily & Virus name (abbreviation) & Natural host & GeneBank accession No. \\
\hline \multirow[t]{45}{*}{ Alphaherpesvirinae } & Suid herpesvirus 1 (SuHV-1) & \multirow[t]{4}{*}{ Sus scrofa (Pig) } & AEM64121 \\
\hline & Pseudorabies virus (PRV) & & AEM64052 \\
\hline & & & AEM63983 \\
\hline & & & AAP82011 \\
\hline & Equid herpesvirus 1(EHV-1) & \multirow[t]{2}{*}{ Equus caballus (Horse) } & YP_053074 \\
\hline & Equine abortion virus (EAV) & & \multirow{3}{*}{ NP_045246 } \\
\hline & Equine herpesvirus 4 (EHV-4) & \multirow[t]{2}{*}{ Equus caballus (Horse) } & \\
\hline & Equine rhinopneumonitis virus (ERV) & & \\
\hline & Equid herpesvirus 9 (EHV-9) & \multirow[t]{2}{*}{ Equus caballus (Horse) } & \multirow[t]{2}{*}{ YP_002333510 } \\
\hline & Gazelle herpesvirus 1 (GHV-1) & & \\
\hline & Felid herpesvirus 1 (FeHV-1) & Felidae (Cat) & YP_003331548 \\
\hline & Bovine herpesvirus 1 (BoHV-1) & \multirow{2}{*}{ Bos taurus (Cattle) } & \multirow{2}{*}{ NP_045327 } \\
\hline & Infectious bovine rhinotracheitis virus (IBRV) & & \\
\hline & Bovine herpesvirus 5 (BoHV-5) & \multirow[t]{2}{*}{ Bos taurus (Cattle) } & \multirow[t]{2}{*}{ YP_003662493 } \\
\hline & Bovine encephalitis herpesvirus (BEHV) & & \\
\hline & Human herpesvirus 3 (HHV-3) & \multirow[t]{2}{*}{ Homo sapiens (Human) } & \multirow[t]{2}{*}{ NP_040150 } \\
\hline & Varicella-zoster virus (VZV) & & \\
\hline & Human herpesvirus 1 (HHV-1) & \multirow[t]{2}{*}{ Homo sapiens (Human) } & \multirow[t]{2}{*}{ ADM23274 } \\
\hline & Herpes simplex virus 1 (HSV-1) & & \\
\hline & Human herpesvirus 2 (HHV-2) & \multirow[t]{2}{*}{ Homo sapiens (Human) } & NP_044501 \\
\hline & Herpes simplex virus 2 (HSV-2) & & \\
\hline & Cercopithecine herpesvirus 1 (CeHV-1) & Macaca mulatta (Monkey) & NP_851891 \\
\hline & Macacine herpesvirus 1 (McHV-1) & & \\
\hline & Monkey B virus & & \\
\hline & Cercopithecine herpesvirus 2 (CeHV-2) & Cercopithecus aethiops (Monkey) & YP_164474 \\
\hline & Simian agent 8 (SA8) & & \\
\hline & Cercopithecine herpesvirus 9 (CeHV-9) & Erythrocebus patas (Monkey) & NP_077442 \\
\hline & Simian varicella virus (SVV) & & \\
\hline & Cercopithecine herpesvirus 16 (CeHV-16) & Papio cynocephalus (Baboons) & YP_443878 \\
\hline & Papiine herpesvirus 2 (PaHV-1) & & \\
\hline & Herpesvirus papio 2 (HVP-2) & & \\
\hline & Saimiriine herpesvirus 1 (SaHV-1) & Saimiri (Squirrel monkeys) & YP_003933808 \\
\hline & Marmoset herpesvirus (MarHV) & & \\
\hline & Gallid herpesvirus 2 (GaHV-2) & Gallus domesticus (Chicken) & AF147806_30 \\
\hline & Marek's disease virus type 1 (MDV-1) & & \\
\hline & Gallid herpesvirus 3 (GaHV-3) & Gallus gallus (Chicken) & NP_066863 \\
\hline & Marek's disease virus type 2 (MDV-2) & & \\
\hline & Meleagrid herpesvirus 1 (MeHV-1) & Meleagris gallopavo (turkey) & NP_073325 \\
\hline & Anatid herpesvirus 1 (AnHV-1) & Anatid species (Duck) & YP_003084390 \\
\hline & Duck enteritis virus (DEV) & & \\
\hline & Psittacid herpesvirus 1 (PsHV-1) & Amazona oratrix (Parrot) & NP_944404 \\
\hline & Pacheco's disease virus (PDV) & & \\
\hline & Gallid herpesvirus 1 (GaHV-1) & White Leghorn (Chicken) & CAJ28933 \\
\hline & Infectious laryngotrach eitis virus (ILTV) & & \\
\hline & Hawaiian green turtle herpesvirus (HGTHV) & Chelonia mydas (Green turtle) & AAU93329 \\
\hline Betaherpesvirinae & Human herpesvirus 5 (HHV-5) & Homo sapiens (Human) & ACZ80140 \\
\hline & Human cytomegalovirus (HCMV) & & \\
\hline & Saimiriine herpesvirus 3 (SaHV-3) & Saimiri sciureus (Squirrel monkey) & YP_004940226 \\
\hline & Squirrel monkey cytomegalovirus (SMCMV) & & \\
\hline & Cercopithecine herpesvirus 5 (CeHV-5) & Cercopithecus aethiops (Vervet monkey) & YP_004936029 \\
\hline & Geen monkey cytomegalovirus (GMCMV) & & \\
\hline & Simian cytomegalovirus (SCMV) & & \\
\hline & Murid herpesvirus 2 (MuHV-2) & Rattus norvegicus (Norway rat) & NP_064159 \\
\hline & Rat cytomegalovirus (RCMV) & & \\
\hline & Murid herpesvirus 1 (MuHV-1) & Mus domesticus (House mice) & CCE56895 \\
\hline & Murine cytomegalovirus (MCMV) & & \\
\hline & Human herpesvirus 7 (HHV-7) & Homo sapiens (Human) & YP_073777 \\
\hline & Human herpesvirus 6 (HHV-6) & Homo sapiens (Human) & NP_042930 \\
\hline & Porcine cytomegalovirus (PCMV) & Sus scrofa (Pig) & AF $268042 \_3$ \\
\hline & Caviid herpesvirus 2 (CavHV-2) & Cavia porcellus (Guinea pig) & YP_002321 246 \\
\hline & Guinea pig cytomegalovirus (GPCMV) & & \\
\hline
\end{tabular}

Continued on next page 


\begin{tabular}{|c|c|c|c|}
\hline Subfamily & Virus name (abbreviation) & Natural host & GeneBank accession No. \\
\hline \multirow[t]{22}{*}{ Gammaherpesvirinae } & Murid herpesvirus 4 (MuHV-4) & Apodemus sylvaticus (Wood mice) & NP_044907 \\
\hline & $\begin{array}{l}\text { Murine gammaherpesvirus } 68 \text { (MuHV-68) } \\
\text { Ateline herpesvirus } 3 \text { (AtHV-3) }\end{array}$ & Ateles (Snider monkey) & NP 048041 \\
\hline & Herpesvirus ateles (HVA) & 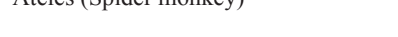 & \\
\hline & $\begin{array}{l}\text { Saimiriine herpesvirus } 2(\mathrm{SaHV}-2) \\
\text { Herpesvirus saimiri (HVS) }\end{array}$ & Saimiri sciureus (Squirrel monkey) & NP_040271 \\
\hline & Bovine herpesvirus 4 (BoHV-4) & Bos taurus (Cattle) & NP_076562 \\
\hline & Human herpesvirus 8 (HHV-8) & Homo sapiens (Human) & YP_001129427 \\
\hline & Kaposi's sarcoma-associated herpesvirus (KSHV) & & \\
\hline & $\begin{array}{l}\text { Cercopithecine herpesvirus } 17 \text { (CeHV-17) } \\
\text { Macacine herpesvirus } 5 \text { (McHV-5) }\end{array}$ & Macaca mulatta (Rhesus monkey) & NP_570817 \\
\hline & Macaca fuscata rhadinovirus (MFRV) & Macaca fuscata (Japanese monkey) & AAT00120 \\
\hline & Ovine herpesvirus 2 (OvHV-2) & Ovis aries (Sheep) & YP_438194 \\
\hline & Alcelaphine herpesvirus 1 (AlHV-1) & Connochaetes taurinus (Wildebeest) & NP_065569 \\
\hline & Malignant catarrhal fever virus (MCFV) & & \\
\hline & Callitrichine herpesvirus 3 (CalHV-3) & Common marmoset (Callithrix jacchus) & NP_733917 \\
\hline & Marmoset lymphocryptovirus (MLCV) & & \\
\hline & Human herpesvirus 4 (HHV-4) & Homo sapiens (Human) & YP_401647 \\
\hline & Epstein-Barr virus (EBV) & & \\
\hline & Cercopithecine herpesvirus 15 (CeHV-15) & Macaca mulatta (Rhesus monkey) & YP_067945 \\
\hline & Macacine herpesvirus 4 (McHV-4) & & \\
\hline & Rhesus lymphocryptovirus (LCV) & & \\
\hline & Equid herpesvirus 2 (EHV-2) & Equus caballus (Horse) & NP_042668 \\
\hline & Cynomys herpesvirus (CyHV) & $\begin{array}{l}\text { Cynomys ludovicianus } \\
\text { (black-tailed prairie dog) }\end{array}$ & ACG63560 \\
\hline & Rodent herpesvirus Peru (RHVP) & Oligoryzomys microtis (pygmy rice rat) & YP 004207906 \\
\hline
\end{tabular}

\section{RESULTS}

\section{PCR amplification and cloning of the PRV UL31 gene}

To isolate the UL31 gene, PCR was carried out using the DNA template from the purified genome of the PRV Becker strain. Although no specific band was amplified from the mock-infected control (Figure 1, lane 1), a target fragment of $816 \mathrm{bp}$, which is consistent with the expected size, was amplified from the purified DNA in PRV-infected PK-15 cells (Figure 1, lane 2). The purified DNA fragment of UL31 was cloned into prokaryotic expression vector pET28a $(+)$ to yield pET28a(+)-UL31 (Figure 1, lane 3), which was confirmed by restriction digestion analysis (Figure 1, lanes 4 and 5), PCR amplification (Figure 1, lane 6), and DNA sequencing (Figure 2A).

\section{Bioinformatic analysis of the PRV UL31 nucleotide sequence}

On the basis of sequencing analysis and ORF Finder analysis, an integrated ORF of PRV UL31 consisting of $816 \mathrm{bp}$ was a hit. Moreover, according to the nucleotide sequence similarity search, three nucleotide sequences (accession Nos. JF797218, JF797217 and AY318876) highly matched with the target nucleotide sequence of the PRV Becker strain UL31 (similarity up to 100, 100 and 98\%, respectively) (Figure 2A), which corresponded to the UL31 gene of PRV Kaplan, Bartha and Ea strain, respectively. Nucleotide sequence multiple alignment of the PRV UL31 gene with its homologous genes of 45 reference herpesviruses demonstrated that PRV UL31 had a remarkably high homology of 52.5 to $61.4 \%$ with the members of the subfamily Alphaherpesvirinae, i.e., equine herpesvirus 4 (EHV-4), EHV-9, EHV-1, human herpesvirus 1 (HHV-1, HSV-1), bovine herpesvirus 5 (BoHV-5), saimiriine 
herpesvirus 1 (SaHV-1), cercopithecine herpesvirus 2 (CeHV-2), CeHV-1, HHV-2 (HSV-2), CeHV-16 and BoHV-1. However, low homology (less than 20\%) was detected between PRV and members of subfamilies Betaherpesvirinae and Gammaherpesvirinae (Table 2).
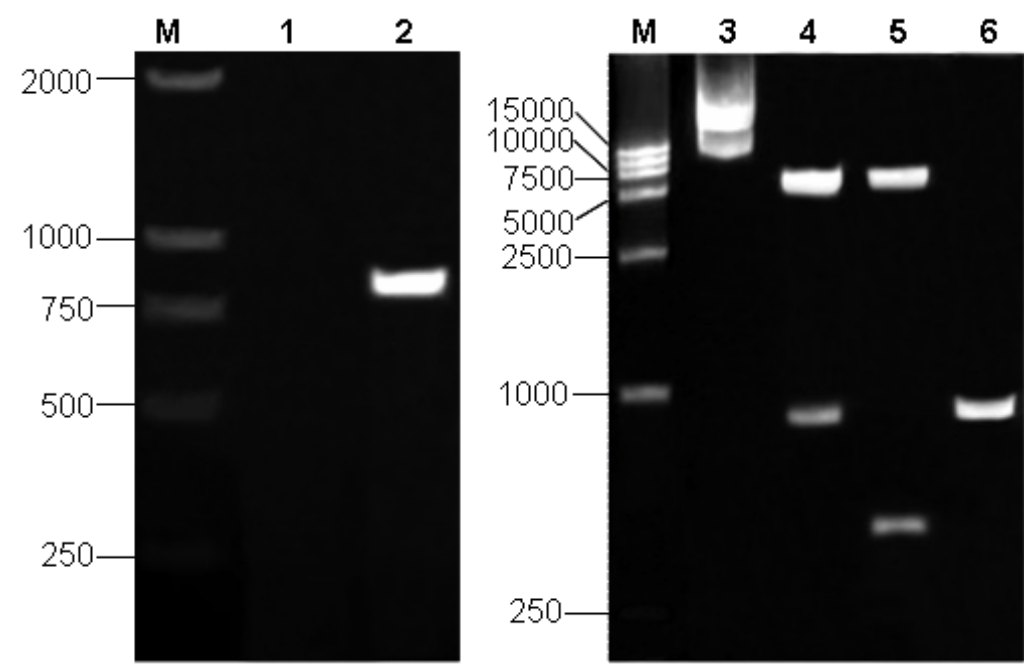

Figure 1. PCR amplification and restriction enzyme analysis of the recombinant plasmid pET28a(+)-UL31. Lanes 1 and 2 = PCR amplification product of the PRV UL31 gene using DNA purified from mock- and PRV-infected PK-15 cells as the template, respectively; lane $3=$ the recombinant plasmid pET28a(+)-UL31; lane $4=$ restriction enzyme digestion product of pET28a(+)-UL31 with EcoRI and HindIII; lane 5 = restriction enzyme digestion product of pET28a(+)-UL31 with EcoRI and XhoI; lane $6=\mathrm{PCR}$ amplification product of the PRV UL31 gene from pET28a $(+)$-UL31. Samples were electrophoresed through a 1\% agarose gel and stained with ethidium bromide. The electrophoresis migration of molecular mass marker (M, TaKaRa) was also shown.

\section{Bioinformatics analysis of PRV UL31 polypeptide sequence}

On the basis of aa sequence similarity search, three aa sequences (accession Nos. AEM64052, AEM63983 and AAP82011) decidedly matched with the target aa sequence of the PRV Becker strain UL31 (similarity up to 100, 100 and 98\%, respectively), which correspond to the UL31 protein of PRV Kaplan, Bartha and Ea strain, respectively (Figure 2B). aa sequence multiple alignment of PRV UL31 with its homologous proteins of 45 reference herpesviruses showed relatively high homology of 50.2 to $60.5 \%$ between PRV UL31 and its counterparts of the subfamily Alphaherpesvirinae of Meleagrid herpesvirus 1 (MeHV-1), CeHV-2, HSV-1, HSV-2, CeHV-16, CeHV-1, SaHV-1, Felid herpesvirus 1 (FeHV-1), CeHV9, VZV, BoHV-1, BoHV-5, EHV-9, EHV-4 and EHV-1. However, PRV UL31 showed no substantial homology (only just 11.7 to $14.9 \%$ ) with UL31-like proteins of the subfamilies Betaherpesvirinae and Gammaherpesvirinae of HHV-6, Murid herpesvirus 1 (MuHV-1), CyHV, EHV-2, HHV-5, SaHV-3, MuHV-2, CeHV-5, BoHV-4, HHV-7, Caviid herpesvirus 2 (CavHV-2) and SaHV-2 (Table 2). Furthermore, conserved domain analysis indicated that PRV UL31 contained a conserved domain PHA03328 superfamily (Figure 2C), which encodes nuclear egress lamina protein UL31. Accordingly, PRV UL31 may have a close relationship with the PHA03328 protein family and may have a high similarity with its counterparts encoded by herpes UL31 genes. Thus, UL31 belonged to the PHA03328 superfamily. 
A

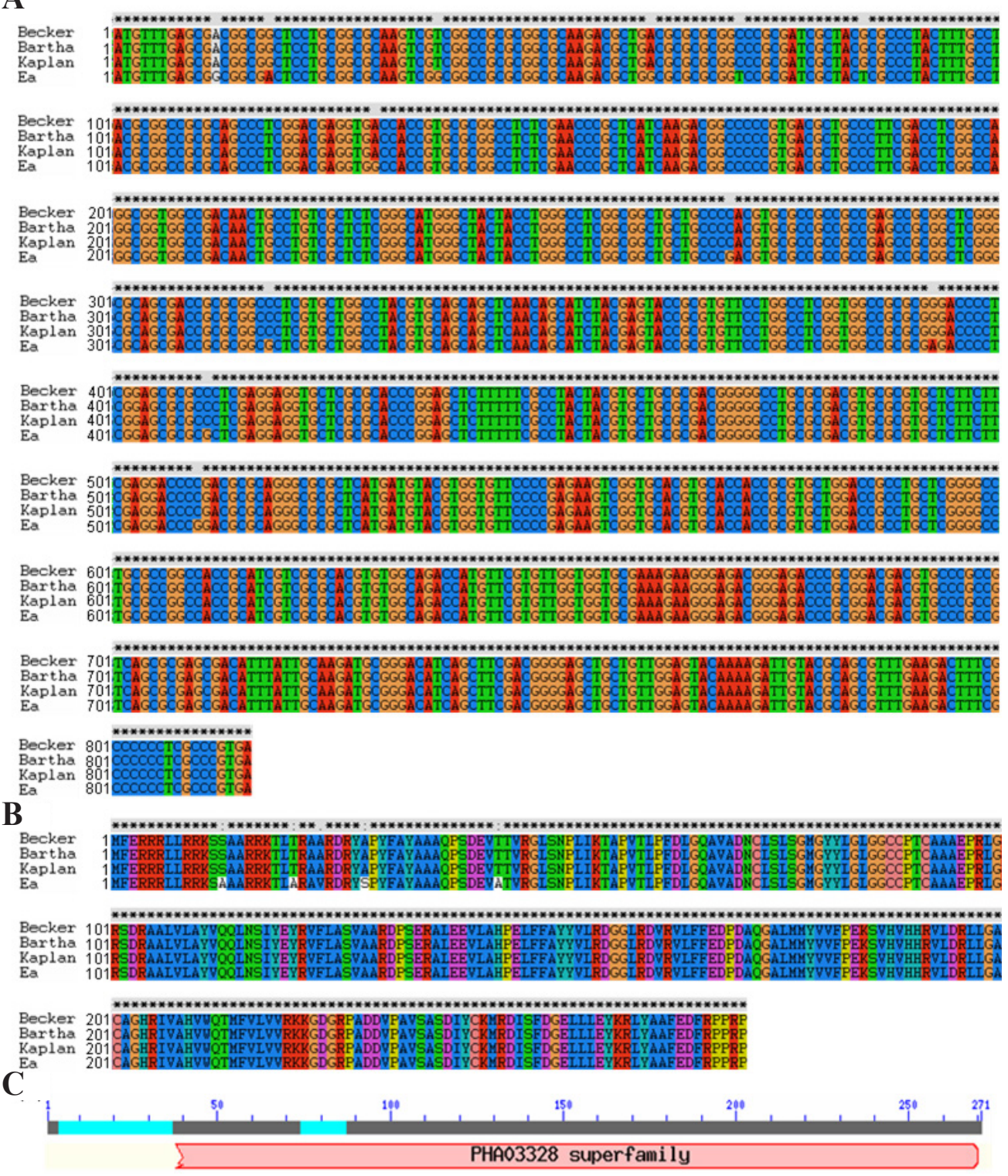

Figure 2. Conserved domain analysis of PRV UL31 and multiple nucleic acid and aa sequence alignments of the UL31 gene of the PRV Becker strain with other PRV strains. A. Multiple nucleic acid sequence alignment of the UL31 gene of the PRV Becker strain (JF797219) with Kaplan (JF797218), Bartha (JF797217) and Ea strain (AY318876) using ClustalX 1.8. Asterisks indicate the identical bases among the UL31 genes of Becker, Kaplan, Bartha, and Ea strains. Without asterisks indicate the different bases among the UL31 genes of Becker, Kaplan, Bartha, and Ea strains. B. Multiple aa sequence alignment of the UL31 protein of PRV Becker strain (AEM64121) with Kaplan (AEM64052), Bartha (AEM63983) and Ea strain (AAP82011) using ClustalX 1.8. Asterisks indicate the identical amino acids among the UL31 proteins of Becker, Kaplan, Bartha, and Ea strains. Colons or point indicate the different amino acids among the UL31 proteins of Becker, Kaplan, Bartha, and Ea strains. C. Conserved domain analysis of PRV UL31 by using NCBI Conserved Domains search tool. 
Table 2. Multiple nucleic acid sequence and amino acid sequence alignments of the PRV UL31 gene with its reference herpesvirus.

\begin{tabular}{|c|c|c|c|c|c|c|c|c|c|}
\hline Virus name & EHV-1 & EHV-4 & EHV-9 & CeHV-9 & FeHV-1 & BoHV-1 & BoHV-5 & HHV-3 & HHV-1 \\
\hline $\mathrm{PNH}^{\mathrm{a}}(\%)$ & 53.3 & 52.5 & 52.7 & 42.9 & 45.6 & 61.4 & 56.6 & 46.6 & 56.2 \\
\hline $\mathrm{PAH}^{\mathrm{b}}(\%)$ & 60.5 & 60.5 & 60.1 & 56.1 & 55.7 & 58.7 & 59.0 & 56.5 & 50.9 \\
\hline Virus name & HHV-2 & CeHV-1 & CeHV-2 & CeHV-16 & SaHV-1 & GaHV-2 & GaHV-3 & MeHV-1 & AnHV-1 \\
\hline $\mathrm{PNH}^{\mathrm{a}}(\%)$ & 59.8 & 59.2 & 59.2 & 60.9 & 58.9 & 41.8 & 45.2 & 41.8 & 43.0 \\
\hline $\mathrm{PAH}^{\mathrm{b}}(\%)$ & 52.0 & 52.8 & 50.9 & 52.4 & 53.1 & 49.4 & 49.4 & 50.2 & 48.7 \\
\hline Virus name & PsHV-1 & GaHV-1 & HGTHV & HHV-5 & SaHV-3 & CeHV-5 & MuHV-2 & MuHV-1 & HHV-7 \\
\hline $\mathrm{PNH}^{\mathrm{a}}(\%)$ & 32.6 & 23.7 & 32.4 & 28.9 & 22.5 & 25.1 & 28.2 & 25.5 & 15.3 \\
\hline $\mathrm{PAH}^{\mathrm{b}}(\%)$ & 24.7 & 24.4 & 25.1 & 14.0 & 14.4 & 14.4 & 14.4 & 12.2 & 14.7 \\
\hline Virus name & HHV-6 & PCMV & CavHV-2 & MuHV-4 & AtHV-3 & SaHV-2 & BoHV-4 & HHV-8 & CeHV-17 \\
\hline $\mathrm{PNH}^{\mathrm{a}}(\%)$ & 19.7 & 22.4 & 25.7 & 22.3 & 19.0 & 17.4 & 18.1 & 22.8 & 22.7 \\
\hline $\mathrm{PAH}^{\mathrm{b}}(\%)$ & 11.7 & 15.3 & 14.8 & 17.3 & 14.9 & 14.9 & 14.4 & 16.6 & 15.1 \\
\hline Virus name & MFRV & OvHV-2 & AlHV-1 & CalHV-3 & HHV-4 & CeHV-15 & EHV-2 & CyHV & RHVP \\
\hline $\mathrm{PNH}^{\mathrm{a}}(\%)$ & 23.7 & 21.1 & 20.3 & 16.5 & 24.0 & 24.0 & 25.2 & 17.9 & 22.2 \\
\hline $\mathrm{PAH}^{\mathrm{b}}(\%)$ & 15.1 & 17.0 & 15.1 & 15.1 & 16.2 & 16.2 & 14.0 & 12.9 & 16.6 \\
\hline
\end{tabular}

aPNH indicates the multiple nucleic acid sequence alignment of the UL31 gene of the PRV Becker strain with its homologous genes of 45 selected species (Table 1) by using the MEGALIGN program in LASERGENE (DNAStar 7.0) with Clustal V Method, and sequence distance was calculated using weight matrix Identity. Gaps had been introduced by the alignment program to maximize the homology. ${ }^{\mathrm{b} A H}$ indicates the multiple aa sequence alignment of UL31 of the PRV Becker strain with its homologous proteins of 45 selected species (Table 1) by using the MEGALIGN program in LASERGENE (DNAStar 7.0) with Clustal V Method, and sequence distance was calculated using weight matrix PAM250. Gaps had been introduced by the alignment program to maximize the homology.

Phylogenetic analysis was performed on the basis of the aa sequences of PRV UL31 and the UL31-like proteins of 45 reference herpesviruses (Table 1). Phylogenetic analysis revealed that they could be preliminarily separated into different subfamilies, i.e., Alphaherpesvirinae, Betaherpesvirinae and Gammaherpesvirinae, which corresponded with the existing classification of the herpesvirus subfamily (Figure 3). Furthermore, the results also showed that PRV UL31 first clustered with members of the subfamily Alphaherpesvirinae of BoHV-1, BoHV-5, EHV-1, EHV-9, EHV-4 and FeHV-1 and then clustered with other members of subfamilies Betaherpesvirinae and Gammaherpesvirinae (Figure 3). Therefore, PRV may have a closer evolutionary relationship with members of the subfamily Alphaherpesvirinae than other members of subfamilies Betaherpesvirinae and Gammaherpesvirinae.

Signal polypeptide prediction indicated that there was no signal polypeptide cleavage site in PRV UL31 (Figure 4A), and that there was no potential transmembrane domain in the polypeptide chain of PRV UL31 (Figure 4B). Moreover, N-linked glycosylation site (Asn-X-Ser/Thr) prediction demonstrated that there was no potential N-glycosylation site in PRV UL31 (Figure 4C), which conversely proved that there was no signal peptide in this protein. Interestingly, there were 12 potential phosphorylation sites in PRV UL31 (Figure 4D), including 9 serine, 1 tyrosine and 2 threonine phosphorylation sites. In addition, the hydrophobicity analysis of PRV UL31 showed that there were 9 main hydrophobic regions located at aa 48-95, 103-115, 120-130, 142-155, 158-164, 169-185, 192-220, 232-240, and 255-259 (Figure 5A), and the proportionality of hydrophilic region was nearly in conformity with the hydrophobic region (Figure 5B). 


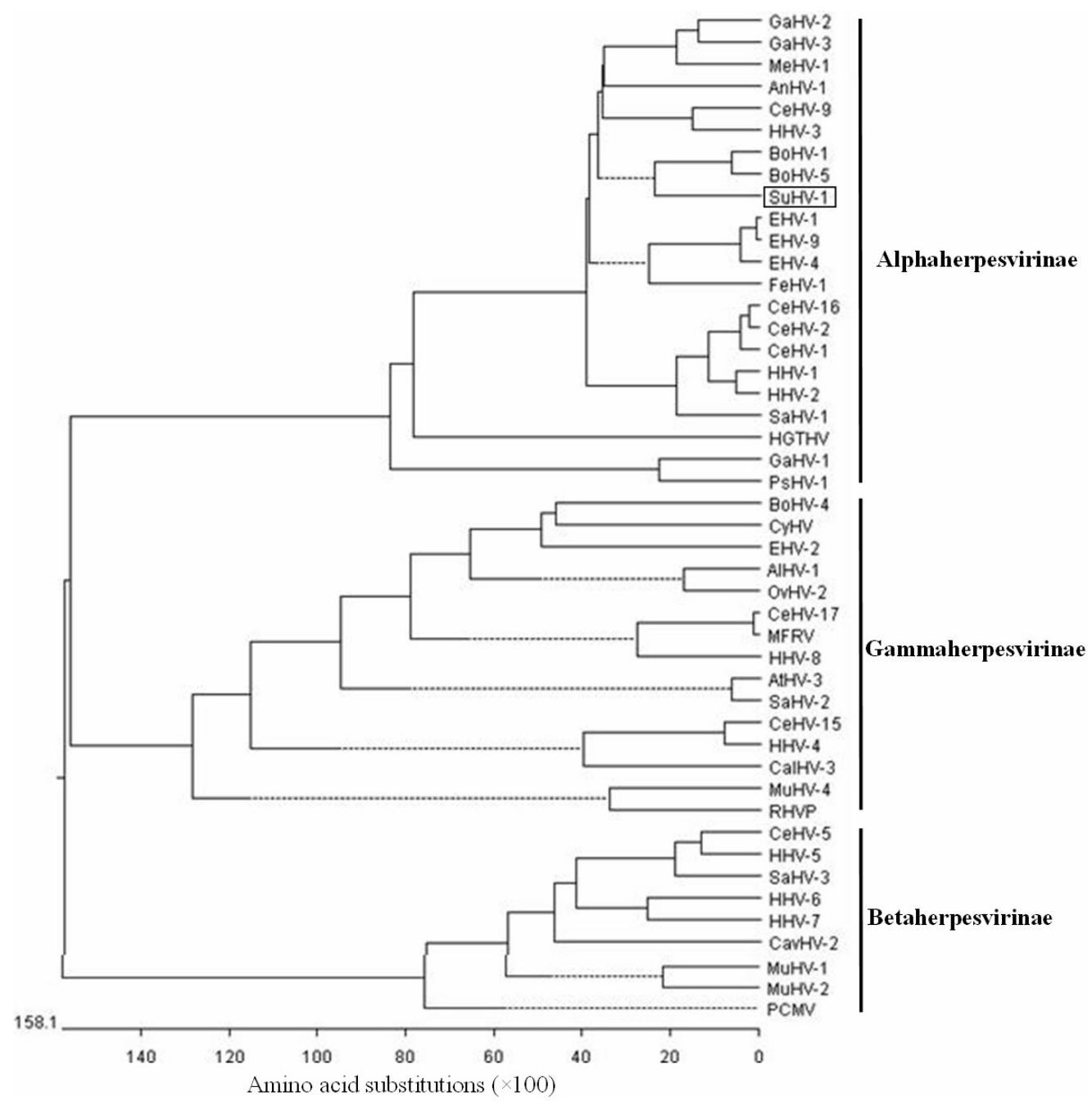

Figure 3. Evolutionary relationships of the putative PRV UL31 protein with its 45 reference herpesviruses from different species (Table 1). Phylogenetic tree of these proteins was generated by using the MEGALIGN program in LASERGENE (DNAStar 7.0) with the Clustal V Method, and sequence distance was calculated using weight matrix PAM250. Gaps had been introduced by the alignment program to maximize the homology.

Analysis of potential B-cell epitope determinant revealed that there were several potential B-cell epitopes in PRV UL31, which were located in or adjacent to aa 1-31, 37-58, 93-106, 129-140, 153-164, 166-174, 183-188, 192-199, 219-259, and 264-271 (Figure 5C). For the secondary structure (Figure 6A), it was shown that PRV UL31 primarily consisted of $\alpha$-helix $(41.33 \%)$ and random coil $(36.53 \%)$ structures, whereas the $\beta$-strand accounted for the smallest component (22.14\%). 3-D structure prediction for PRV UL31 showed that a known 3-D structure model with a relatively high homology with UL31 was found (Figure $6 \mathrm{~B})$, which was predominantly composed of $\alpha$-helix and $\beta$-turn structures. 

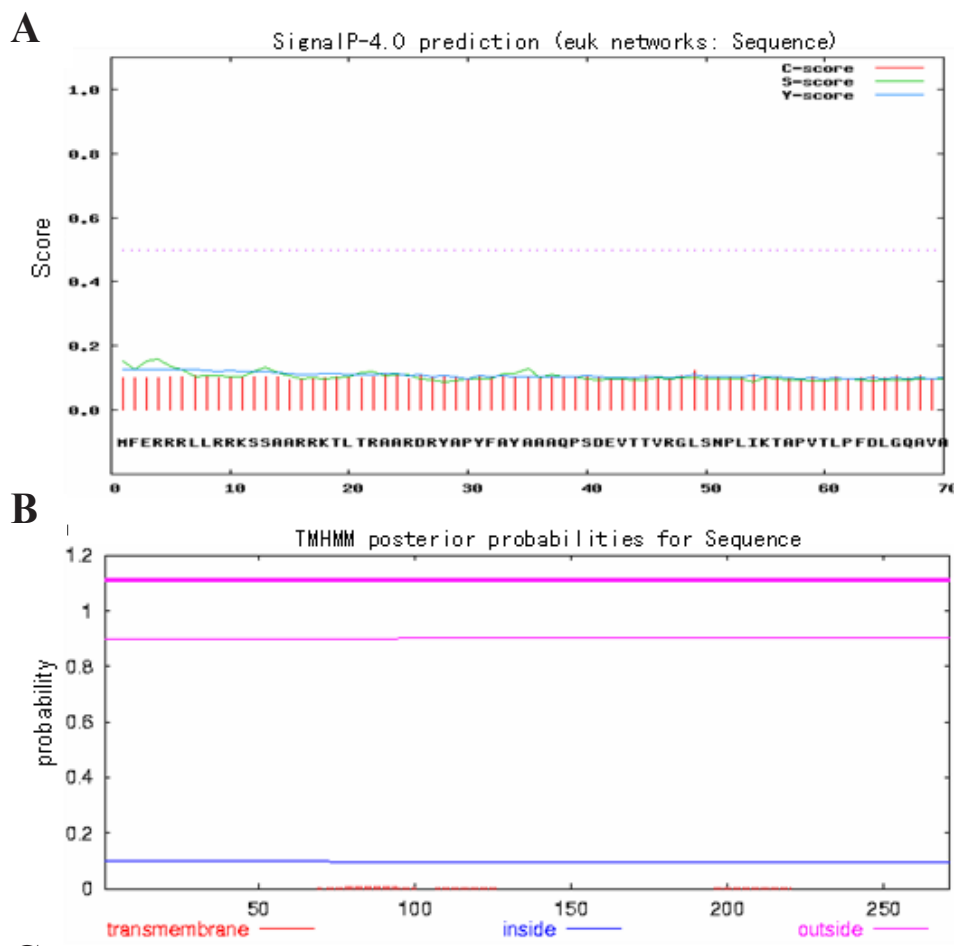

$\mathbf{C}$

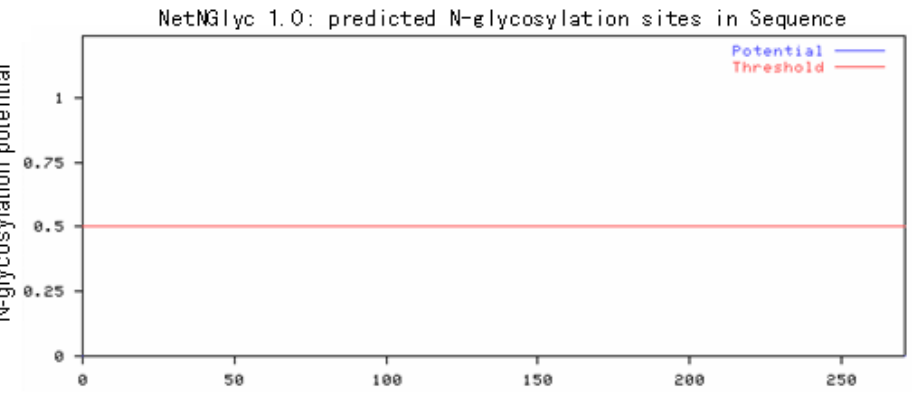

D

NetPhos 2.0: predicted phosphorylation sites in Sequence

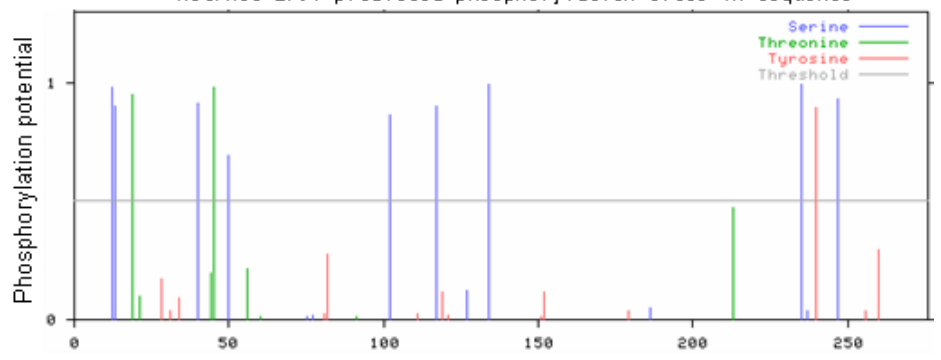

Figure 4. Prediction of signal peptide sequence, transmembrane domain, glycosylation site, and phosphorylation site of PRV UL31. Signal peptide sequence, transmembrane domain, glycosylation site, and phosphorylation site of PRV UL31 were analyzed by SignalP-4.0 Server (A), TMHMM program (B), NetNGlyc 1.0 program (C) and NetPhos 2.0 program $(\mathbf{D})$, respectively. 

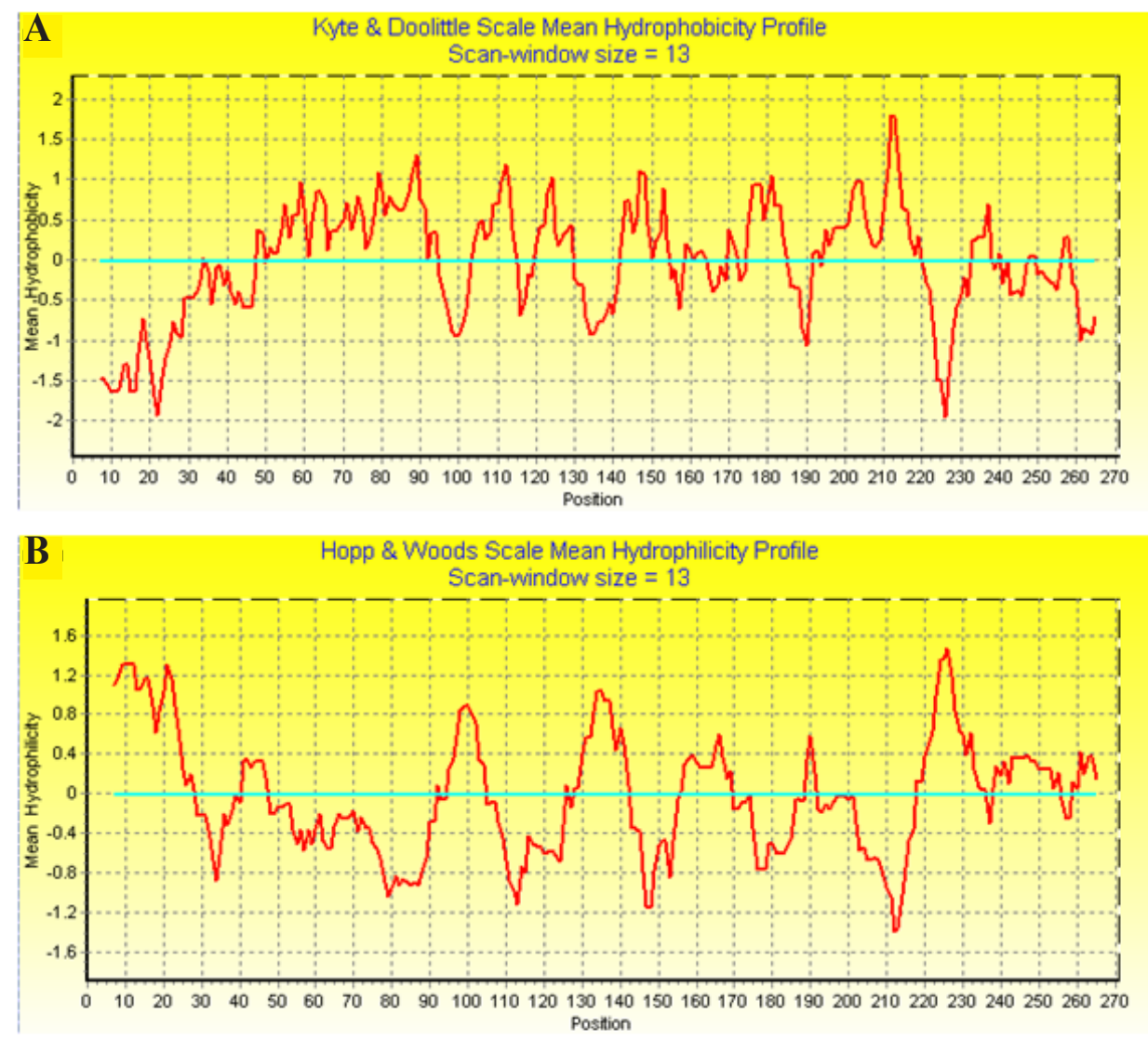

C

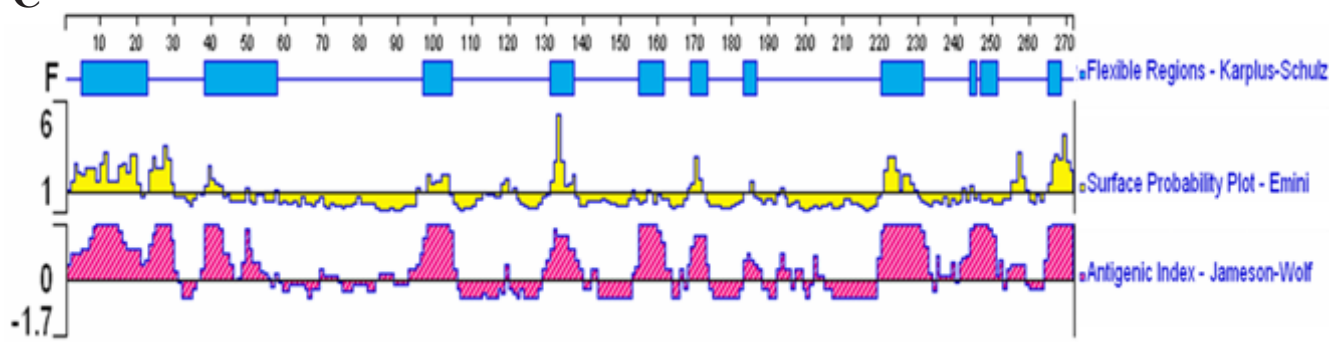

Figure 5. Hydrophobicity, hydrophilicity and antigenic analyses of PRV UL31. The hydrophobicity (A) or hydrophilicity profile (B) of PRV UL31 was determined with the values of Kyte and Doolittle (1982) or Hopp and Woods (1981) by using a 13-amino acid window. The upwardpointing peaks represent the most hydrophobic regions (A) and the most hydrophilic regions (B). C. Antigenic analysis of PRV UL31 was carried out by the PROTEAN software of DNAStar based on its flexibility, surface probability and antigenic index by the determination of its primary structure. 
A

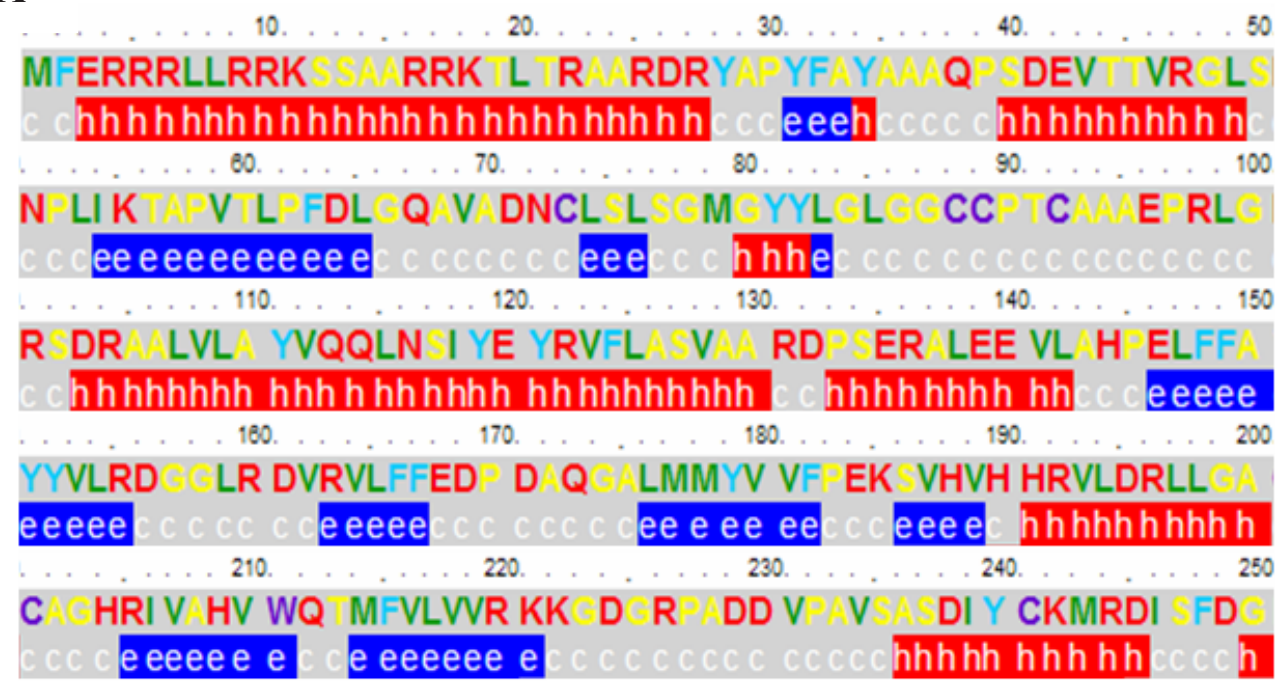

$280 \ldots \ldots 270$

\section{ELLLEYKRLY A FEDF R}

\section{hhhhhhhhhh hhhh}

B

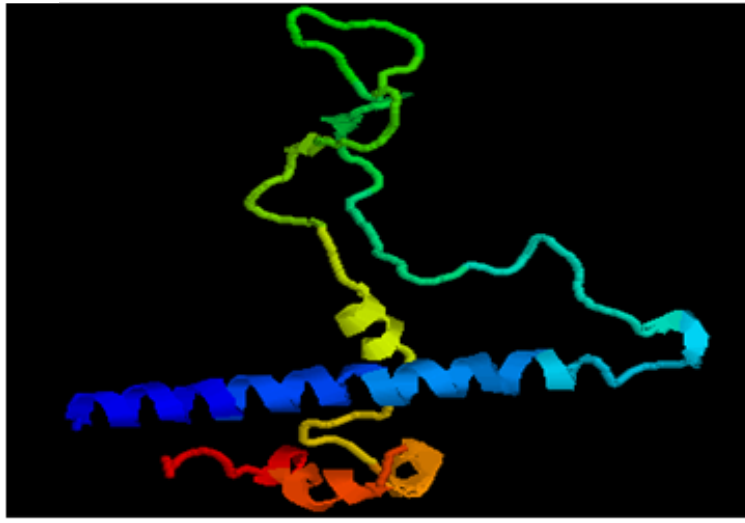

Number of H-Bonds......100

Number of Helices........6

Number of Strands...... 3

Number of Turns..........17

Figure 6. Secondary structure and three-dimension (3-D) structure prediction of PRV UL31. A. Secondary structure of PRV UL31. The secondary structure was predicted by using the PSIpred program and the letters $h, e$, and c indicate alpha helix, extended (beta strand) and coil, respectively. B. 3-D structure of PRV UL31. The 3-D structure was predicted by employing the protein modeling server database CPHmodels 3.2 Server. The number of H-Bond, Helice, Strand, and Turn included in this model were 100, 6, 3 and 17, respectively.

\section{DISCUSSION}

According to PCR amplification, cloning and sequencing, evidence of the UL31 gene of the PRV Becker strain was confirmed. Bioinformatic analysis indicated that its encoded protein UL31 is a member of the PHA03328 superfamily, which encodes nuclear egress lam- 
ina protein UL31 and is conserved throughout the herpesvirus family. Although the biological properties of most of the herpesviral UL31 homologues have been poorly understood until now, a common feature is the interaction of UL31 and UL34 and their colocalization at the nuclear envelope or nuclear rim occurring in distinct herpesvirus subfamilies, such as alphaherpesvirus PRV (Fuchs et al., 2002), HSV-1 (Reynolds et al., 2001) and HSV-2 (Yamauchi et al., 2001), betaherpesvirus murine cytomegalovirus (Lotzerich et al., 2006) and gammaherpesvirus EBV (Lake and Hutt-Fletcher, 2004) and Kaposi's sarcoma-associated herpesvirus (Santarelli et al., 2008). Another interesting property is their importance for primary envelopment and nuclear egress in all herpesvirus subfamily (Reynolds et al., 2001; Fuchs et al., 2002; Gonnella et al., 2005; Miller et al., 2010). Besides, PRV UL31 is essential for neurovirulence in mice (Klopfleisch et al., 2006), although it is nonessential for PRV infection in respiratory epithelial cells.

In the course of evolution, a virus is generally conservative and only few genes will undergo mutation. Thus, the viral evolution can be investigated at the molecular level. According to the analysis results (Figure 2A and B), the nucleotide sequence similarity of UL31 of the PRV Becker strain (JF797219) with Ea (AY318876), Kaplan (JF797218) and Bartha (JF797217) strain were perfectly matched and up to 98 to $100 \%$. Moreover, in agreement with nucleotide sequence similarity, their aa sequence similarity was also extremely matched and up to the same level. Therefore, these results revealed a close relationship between different PRV strains. It is well known that the mutation of a virus may occur in response to environmental stress. Therefore, it might have been the small mutation of the Ea strain that endowed the variance of virulence of PRV, since the Ea strain was derived from the host of various regions when compared with other three PRV strains. Furthermore, multiple nucleic acid sequence and aa sequence alignments of PRV UL31 and the UL31-like proteins showed that PRV UL31 had a relatively high homology with members of the subfamily Alphaherpesvirinae, especially BoHV-1 (61.4\%) and EHV-1 (60.5\%), respectively. Thus, it can be concluded that the PRV UL31 has a relatively high homology with the subfamily Alphaherpesvirinae but not the subfamily Betaherpesvirinae or Gammaherpesvirinae. Besides, phylogenetic analysis clearly demonstrated that PRV belonged to the subfamily Alphaherpesvirinae, which was consistent with a previous report (McGeoch et al., 2000). The phylogenetic tree also showed that PRV UL31 had a closer evolutionary relationship with the member of the subfamily Alphaherpesvirinae than other members of subfamilies Betaherpesvirinae and Gammaherpesvirinae. Accordingly, these results may provide useful insight for exploring the origin and evolution of the herpesviral UL31 gene and perhaps other herpesviral genes.

Phosphorylation site prediction showed that there were 12 potential phosphorylation sites in PRV UL31, including 9 serine, 1 tyrosine and 2 threonine phosphorylation sites. It has been reported that serine phosphorylation of HSV-1 UL31 by US3 is essential for the normal function of UL31 protein, which is involved in modulating the proper localization of pUL31/ pUL34 and formation of primary envelopment and the subsequent fusion of the nascent virion envelope with the outer nuclear membrane (Kato et al., 2006; Mou et al., 2009). HSV-1 UL31 can also be phosphorylated by US3.5 kinase, whereas the phosphorylation sites and the function of this phosphorylation are still not known (Kato et al., 2006). Thus, the phosphorylation of PRV UL31 may also play an important role during PRV infection, perhaps in modulating its proper localization or other uncharacterized functions, such as potential regulatory role in the localization of the nuclear envelopment complex and the egress of nucleocapsids. 
Nevertheless, hydrophobicity and hydrophilicity analyses indicated that the proportion of hydrophobic region was almost in consonance with the hydrophilic region existing in PRV UL31, which could represent the internal and surface regions of the protein. Furthermore, no potential transmembrane domain, signal peptide and N-linked glycosylation site (Asn$\mathrm{X}$-Ser/Thr) was present in PRV UL31, suggesting that this protein may be a true tegument protein, but not a glycoprotein (Granzow et al., 2004). Secondary structure prediction revealed that PRV UL31 predominantly contains $\alpha$-helix and random coil structures, whereas its 3-D conformation mainly consisted of extended $\alpha$-helices linked by $\beta$-turns. Therefore, the $\alpha$-helix principal components of PRV UL31, which contains many highly conserved residues, may be engaged in its self-association or the interactions with other constituents of the tegument (Benach et al., 2007), nucleocapsid (Benach et al., 2007; Ohta et al., 2011) and the host proteins (Uesugi et al., 1997), whereas the $\beta$-turn might play a fundamental role in its stabilization.

As more information becomes available on protein antigens, it should be possible to use this information to predict the locations of antigenic determinants before any immunological testing is carried out. However, the elucidation of protein antigenic structures is presently a difficult, uncertain and time-consuming task. Earlier methods are based on the assumption that the antigenic region is primarily the hydrophilic region at the surface of the protein molecule (Hopp and Woods, 1981; Welling et al., 1985), but there has been some inaccuracy and limitation about it. To improve the accuracy, the B-cell epitopes of PRV UL31 were predicted using DNAStar PROTEAN programs, on the basis of flexibility, antigenic index and surface probability by the determination of its primary structure. We suggest that improved knowledge of the antigenic and structural properties of PRV UL31 resulting from this study could lead to methods for developing new antibodies and immunoassays for application in the clinical diagnosis of PRV.

In conclusion, we describe here the cloning and molecular characterization of the PRV UL31 gene. Elucidating the relationship between molecular characterization and genetic evolution of PRV UL31 gene will contribute to our understanding of this virus at the molecular level and also enrich the herpesvirus database. This study also provides some insight for further research on the function and mechanism of the UL31 gene during PRV infection.

\section{ACKNOWLEDGMENTS}

Research supported by the Foundation for Distinguished Young Talents in Higher Education of Guangdong, China (\#2013LYM_0096); the Science and Technology New Star in Zhu Jiang, Guangzhou City (\#2013J2200018); the Natural Science Foundation of Guangdong Province (\#S2013040016596); the National Natural Science Foundation of China (\#31200120); the First Batch of Young Core Instructor of Guangzhou Medical University and the Students' Extracurricular Scientific and Technological Activities in Guangzhou Medical University (\#2013A003). We thank Dr. Lynn W. Enquist for the generous gift of pBecker2.

\section{REFERENCES}

Altschul SF, Madden TL, Schaffer AA, Zhang J, et al. (1997). Gapped BLAST and PSI-BLAST: a new generation of protein database search programs. Nucleic Acids Res. 25: 3389-3402.

Benach J, Wang L, Chen Y, Ho CK, et al. (2007). Structural and functional studies of the abundant tegument protein ORF52 from murine gammaherpesvirus 68. J. Biol. Chem. 282: 31534-31541. 
Bendtsen JD, Nielsen H, von Heijne G and Brunak S (2004). Improved prediction of signal peptides: SignalP 3.0. J. Mol. Biol. 340: 783-795.

Blom N, Gammeltoft S and Brunak S (1999). Sequence and structure-based prediction of eukaryotic protein phosphorylation sites. J. Mol. Biol. 294: 1351-1362.

Burland TG (2000). DNASTAR's Lasergene sequence analysis software. Methods Mol. Biol. 132: 71-91.

Chang YE and Roizman B (1993). The product of the UL31 gene of herpes simplex virus 1 is a nuclear phosphoprotein which partitions with the nuclear matrix. J. Virol. 67: 6348-6356.

Fuchs W, Klupp BG, Granzow H, Osterrieder N, et al. (2002). The interacting UL31 and UL34 gene products of pseudorabies virus are involved in egress from the host-cell nucleus and represent components of primary enveloped but not mature virions. J. Virol. 76: 364-378.

Gonnella R, Farina A, Santarelli R, Raffa S, et al. (2005). Characterization and intracellular localization of the EpsteinBarr virus protein BFLF2: interactions with BFRF1 and with the nuclear lamina. J. Virol. 79: 3713-3727.

Granato M, Feederle R, Farina A, Gonnella R, et al. (2008). Deletion of Epstein-Barr virus BFLF2 leads to impaired viral DNA packaging and primary egress as well as to the production of defective viral particles. J. Virol. 82: 4042-4051.

Granzow H, Klupp BG and Mettenleiter TC (2004). The pseudorabies virus US3 protein is a component of primary and of mature virions. J. Virol. 78: 1314-1323.

Hopp TP and Woods KR (1981). Prediction of protein antigenic determinants from amino acid sequences. Proc. Natl. Acad. Sci. U. S. A. 78: 3824-3828.

Jones DT (1999). Protein secondary structure prediction based on position-specific scoring matrices. J. Mol. Biol. 292: 195-202.

Kato A, Yamamoto M, Ohno T, Tanaka M, et al. (2006). Herpes simplex virus 1-encoded protein kinase UL13 phosphorylates viral Us3 protein kinase and regulates nuclear localization of viral envelopment factors UL34 and UL31. J. Virol. 80: 1476-1486.

Klopfleisch R, Klupp BG, Fuchs W, Kopp M, et al. (2006). Influence of pseudorabies virus proteins on neuroinvasion and neurovirulence in mice. J. Virol. 80: 5571-5576.

Kyte J and Doolittle RF (1982). A simple method for displaying the hydropathic character of a protein. J. Mol. Biol. 157: 105-132.

Lake CM and Hutt-Fletcher LM (2004). The Epstein-Barr virus BFRF1 and BFLF2 proteins interact and coexpression alters their cellular localization. Virology 320: 99-106.

Li M, Wang S, Cai M, Guo H, et al. (2011a). Characterization of molecular determinants for nucleocytoplasmic shuttling of PRV UL54. Virology 417: 385-393.

Li M, Wang S, Cai M and Zheng C (2011b). Identification of nuclear and nucleolar localization signals of pseudorabies virus (PRV) early protein UL54 reveals that its nuclear targeting is required for efficient production of PRV. J. Virol. 85: 10239-10251.

Li M, Zhao Z, Chen J, Wang B, et al. (2012). Characterization of synonymous codon usage bias in the pseudorabies virus US1 gene. Virol. Sin. 27: 303-315.

Liang L and Baines JD (2005). Identification of an essential domain in the herpes simplex virus 1 UL34 protein that is necessary and sufficient to interact with UL31 protein. J. Virol. 79: 3797-3806.

Lotzerich M, Ruzsics Z and Koszinowski UH (2006). Functional domains of murine cytomegalovirus nuclear egress protein M53/p38. J. Virol. 80: 73-84.

Marchler-Bauer A, Anderson JB, Cherukuri PF, DeWeese-Scott C, et al. (2005). CDD: a Conserved Domain Database for protein classification. Nucleic Acids Res. 33: D192-D196.

McGeoch DJ, Dolan A and Ralph AC (2000). Toward a comprehensive phylogeny for mammalian and avian herpesviruses. J. Virol. 74: 10401-10406.

Miller MS, Furlong WE, Pennell L, Geadah M, et al. (2010). RASCAL is a new human cytomegalovirus-encoded protein that localizes to the nuclear lamina and in cytoplasmic vesicles at late times postinfection. J. Virol. 84: 6483-6496.

Moller S, Croning MD and Apweiler R (2001). Evaluation of methods for the prediction of membrane spanning regions. Bioinformatics 17: 646-653.

Mou F, Wills E and Baines JD (2009). Phosphorylation of the U(L)31 protein of herpes simplex virus 1 by the U(S)3encoded kinase regulates localization of the nuclear envelopment complex and egress of nucleocapsids. J. Virol. 83: 5181-5191.

Nielsen H, Engelbrecht J, Brunak S and von Heijne G (1997). Identification of prokaryotic and eukaryotic signal peptides and prediction of their cleavage sites. Protein Eng. 10: 1-6.

Ohta A, Yamauchi Y, Muto Y, Kimura H, et al. (2011). Herpes simplex virus type 1 UL14 tegument protein regulates intracellular compartmentalization of major tegument protein VP16. Virol. J. 8: 365.

Pomeranz LE, Reynolds AE and Hengartner CJ (2005). Molecular biology of pseudorabies virus: impact on neurovirology 
and veterinary medicine. Microbiol. Mol. Biol. Rev. 69: 462-500.

Reynolds AE, Ryckman BJ, Baines JD, Zhou Y, et al. (2001). U(L)31 and U(L)34 proteins of herpes simplex virus type 1 form a complex that accumulates at the nuclear rim and is required for envelopment of nucleocapsids. $J$. Virol. 75 : 8803-8817.

Roberts KL and Baines JD (2011). UL31 of herpes simplex virus 1 is necessary for optimal NF-kappaB activation and expression of viral gene products. J. Virol. 85: 4947-4953.

Santarelli R, Farina A, Granato M, Gonnella R, et al. (2008). Identification and characterization of the product encoded by ORF69 of Kaposi's sarcoma-associated herpesvirus. J. Virol. 82: 4562-4572.

Simpson-Holley M, Baines J, Roller R and Knipe DM (2004). Herpes simplex virus 1 U(L)31 and U(L)34 gene products promote the late maturation of viral replication compartments to the nuclear periphery. J. Virol. 78: 5591-5600.

Smith GA and Enquist LW (2000). A self-recombining bacterial artificial chromosome and its application for analysis of herpesvirus pathogenesis. Proc. Natl. Acad. Sci. U. S. A. 97: 4873-4878.

Thompson JD, Gibson TJ, Plewniak F, Jeanmougin F, et al. (1997). The CLUSTAL_X windows interface: flexible strategies for multiple sequence alignment aided by quality analysis tools. Nucleic Acids Res. 25: 4876-4882.

Uesugi M, Nyanguile O, Lu H, Levine AJ, et al. (1997). Induced alpha helix in the VP16 activation domain upon binding to a human TAF. Science 277: 1310-1313.

Van Regenmortel MH (1992). Protein antigenicity. Mol. Biol. Rep. 16: 133-138.

Welling GW, Weijer WJ, van der Zee R and Welling-Wester S (1985). Prediction of sequential antigenic regions in proteins. FEBS Lett. 188: 215-218.

Yamauchi Y, Shiba C, Goshima F, Nawa A, et al. (2001). Herpes simplex virus type 2 UL34 protein requires UL31 protein for its relocation to the internal nuclear membrane in transfected cells. J. Gen. Virol. 82: 1423-1428.

Yang K and Baines JD (2011). Selection of HSV capsids for envelopment involves interaction between capsid surface components pUL31, pUL17, and pUL25. Proc. Natl. Acad. Sci. U. S. A. 108: 14276-14281.

Ye GJ and Roizman B (2000). The essential protein encoded by the UL31 gene of herpes simplex virus 1 depends for its stability on the presence of UL34 protein. Proc. Natl. Acad. Sci. U. S. A. 97: 11002-11007. 